\title{
Article \\ Two-Dimensional Digital Beam Steering Based on Liquid Crystal Phase Gratings
}

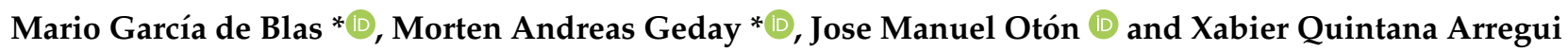 \\ CEMDATIC, ETSI Telecomunicación, Universidad Politécnica de Madrid, Av. Complutense 30, \\ 28040 Madrid, Spain; jm.oton@upm.es (J.M.O.); x.quintana@upm.es (X.Q.A.) \\ * Correspondence: mario.gdeblas@upm.es (M.G.d.B.); morten.geday@upm.es (M.A.G.)
}

check for updates

Citation: García de Blas, M.; Geday, M.A.; Otón, J.M.; Quintana Arregui, X. Two-Dimensional Digital Beam Steering Based on Liquid Crystal Phase Gratings. Appl. Sci. 2021, 11, 3632. https://doi.org/10.3390/ app11083632

Academic Editor: Ye Zhi Ting

Received: 24 March 2021

Accepted: 13 April 2021

Published: 17 April 2021

Publisher's Note: MDPI stays neutral with regard to jurisdictional claims in published maps and institutional affiliations.

Copyright: (c) 2021 by the authors. Licensee MDPI, Basel, Switzerland. This article is an open access article distributed under the terms and conditions of the Creative Commons Attribution (CC BY) license (https:// creativecommons.org/licenses/by/ $4.0 /)$.

\begin{abstract}
Electrically tunable phase gratings are able to steer an incoming light beam without employing movable parts. Here, we present the design and implementation of a 2D beam steering device by cascading two orthogonal 1D liquid crystal (LC) based phase gratings, each having an array of 72 rectangular individually controlled pixels and driven by a custom 12-bit PulseWidth Modulation (PWM) electrical driver. High-resolution structures in glass wafers coated with transparent Indium-Tin Oxide (ITO) have been prepared using Direct Laser Writing (DLW) techniques. With DLW, a high number of pixels can easily be drawn with an interpixel space of less than $3 \mu \mathrm{m}$, leading to devices with a high fill factor. The active area of the cascaded device is $1.1 \times 1.1 \mathrm{~mm}^{2}$. We present a $72 \times 72$ point efficiency map corresponding to a maximum diagonal steering angle of $1.65^{\circ}$. Special attention has been paid to make the device compatible with space application by avoiding electronics in the active area.
\end{abstract}

Keywords: beam steering; liquid crystal; blaze grating; direct laser witting

\section{Introduction}

Pointing a laser beam accurately is relevant for many applications, such as optical tweezers [1], free space optical communications [2], and light detection and ranging [3-5]. To achieve this control, beam steering (BS) systems have been developed, both mechanical and non-mechanical [6].

There is a plethora of mechanical methods where movable micro-mirrors or microlenses steer a light beam by using piezo-actuators, rotating prisms, or microelectromechanical systems [7-9]. The main disadvantage of all these mechanical systems is that they need continuous adjustments to maintain alignment, and they can suffer from external noise [10]. Additionally, micromechanical systems with movable parts are scarcely acceptable for space applications because the harsh conditions during take-off and orbital steps easily jeopardize their stability and proper functioning.

Non-mechanical methods are widely used nowadays, such as acousto-optic and electro-optic deflectors [11,12] and liquid crystal spatial (LC) light modulators [13,14]. When compared to mechanical devices, non-mechanical devices offer a higher reliability, which makes them suitable for space applications [15]. For these devices, large steerable angles and large apertures are desirable.

LCs have been demonstrated to be a good candidate to implement non-mechanical beam steering devices due to their optical properties and swift response to moderate external electric fields. Optical Phased Arrays [16], Pancharatnam-Berry [17], Liquid Crystal on Silicon (LCoS) systems [18,19], and Polarization Gratings [20-22] are some solutions provided by LCs. LC devices are interesting when easy electronic driving, small size, light weight, non-mechanical parts, low power consumption, and low cost are considered as relevant requirements of the intended device.

LC beam steerers are usually prepared as phase-only devices. They are optical systems that interact with light, only modifying their phase and leaving other characteristics, such 
as wavelength or intensity, unchanged $[23,24]$. The aim of this work is to present a high fill factor LC BS device, capable of redirecting an impinging light beam in two dimensions (Figure 1). The steering angle is electronically tunable by spatially varying the retardation pattern of the liquid crystal.

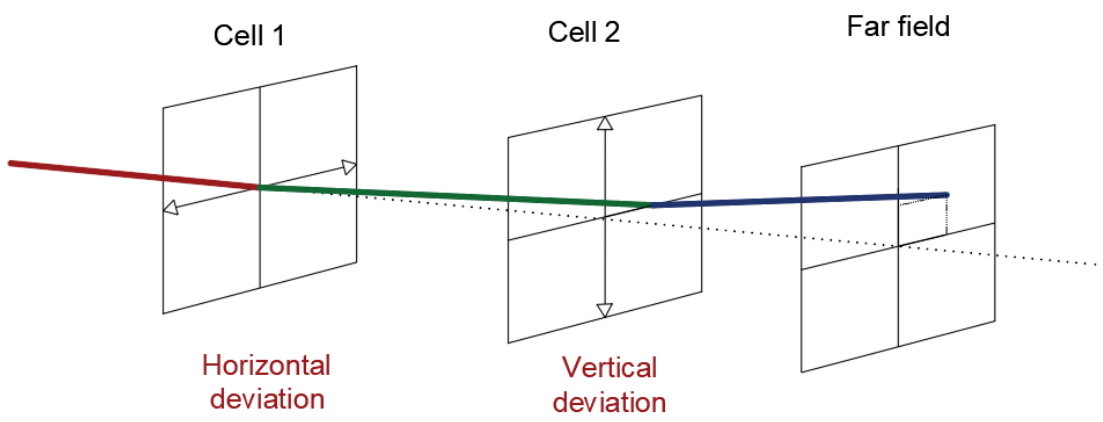

Figure 1. The combination of two 1D LC cells generating a 2D deviation in the incident angle.

\section{Materials and Methods}

\subsection{Beam Steerers for Space Applications}

Several LC structures can be used as beam steerers. Those intended to be applied to space environments need to account for resilience under ionizing radiation conditions. This usually excludes any LC device in which the active area includes electronics, such as the LCoS or the ubiquitous active-matrix thin-film transistor (AM-TFT). In both cases, LCoS and AM-TFT, electronics cannot be adequately shielded from ionizing radiation because the beam steerer must have visibility to outer space.

Passive-driven transmissive systems present a good alternative. The reliability of LC materials under ionizing radiation has already been tested [2]. In passive addressing mode, driving electronics are outside the steering cell, allowing the drivers to be adequately shielded.

The operation principle of this passive-addressed BS is based on phase diffraction gratings. Diffraction gratings are made of a set of linear parallel elements with a pitch comparable to the light wavelength [25]. Diffractive gratings are manufactured using different methods, as recently reviewed in [26].

The device presented in this work is driven digitally (Figure 2). Analog tunable beam steerers can be achieved using high resistivity electrodes [27], albeit with a limited scanning range. The transmissive device is a blaze-grating, i.e., a saw-tooth refractive index profile where every tooth is discretized in a number of steps by setting the effective refractive index of the liquid crystal, pixel by pixel $[28,29]$. Thus, as the light passes through the diffractive structure, it is deviated by an angle.

The diffraction efficiency is the relationship between the diffracted power and the input power. The larger the number of pixels per tooth, the better approximation to the continuous wedge profile, and the higher the diffraction efficiency, at a cost of reduced deviation angle. Fringing fields and crosstalk have been reported to cause a significant loss in efficiency $[30,31]$. 


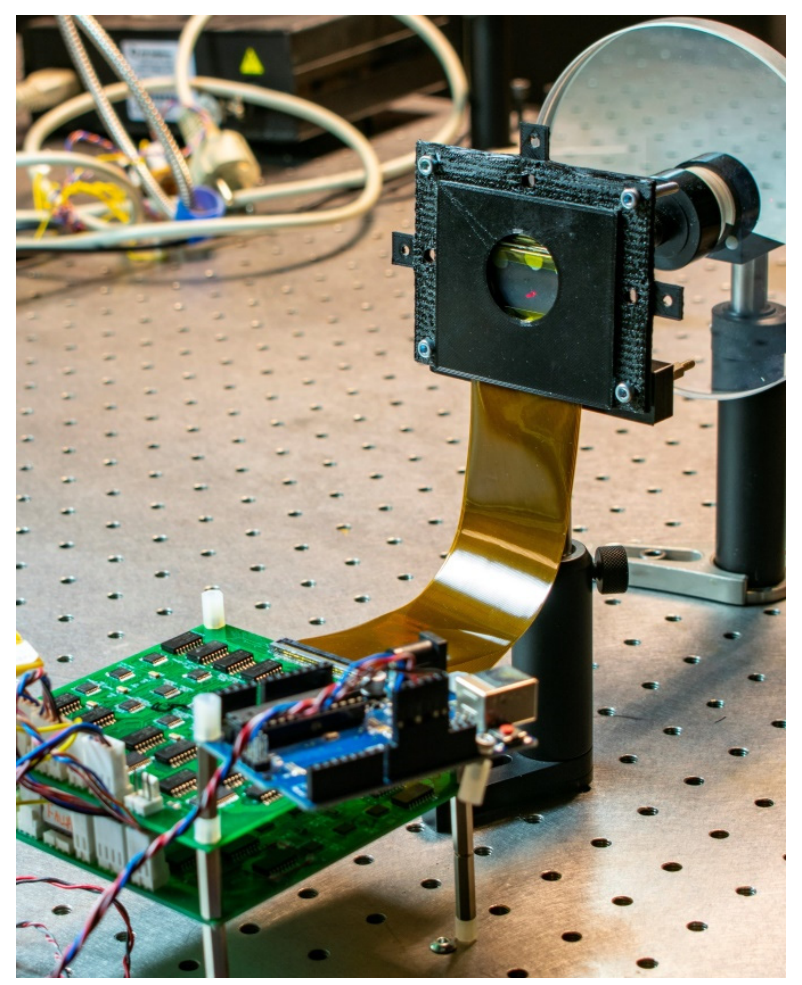

Figure 2. LC beam steering device. The LC cells are in their holder, with the electronic driver. The variable attenuator employed in the setup is also visible.

\subsection{Device Characteristics}

The active area is defined by the pixel width and the total number of individual pixels. The employed nematic LC requires that the incoming light must be polarized. The birefringence of the LC delay the light polarized along the switching plane. The delay, $\delta(V)$, can be calibrated by measuring the interference between the light polarized along the ordinary and the extraordinary axes, respectively, using crossed polarizers as a function of the applied voltage [23]:

$$
\delta(V)=\frac{2 \pi}{\lambda} \cdot\left[n_{e f f}(V)-n_{o}\right] \cdot d,
$$

where $\lambda$ is the wavelenght of the incoming light, $\mathrm{d}$ is the thickness of the LC cell, and $n_{e f f}(V)-n_{o}$ is the effective birefrigence of the LC at a voltage, $\mathrm{V}$.

The intensity variation follows the relationship in Equation (2):

$$
I \propto \sin ^{2} \cdot \frac{\delta(V)}{2}
$$

In the diffractive phase graing, the continously varying phase is "wrapped" between $0 \pi$ and $2 \pi$ [32] (Figure 3). When the pattern corresponds to an integer number of pixels, a recurrent phase delay structure is observed along the grating. In this paper, the term "topology" is used to describe the total retardation of an equivalent wedge prism in which the maxima is $t \cdot 2 \pi$; $t$ being the topology number. The developed driver, which allows for individual addresing in the LC cell, means that the topology can be any fractional number. As an arbitrary convention, the positive sign of the topology has been asigned to deviation to the right and up along the two axes, respectively; Figure 4. 


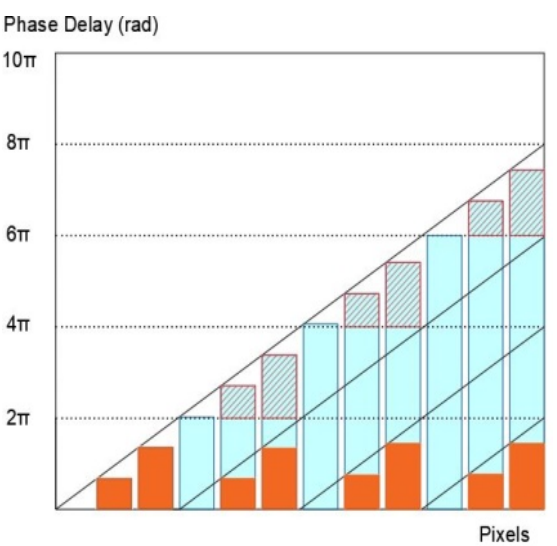

(a)

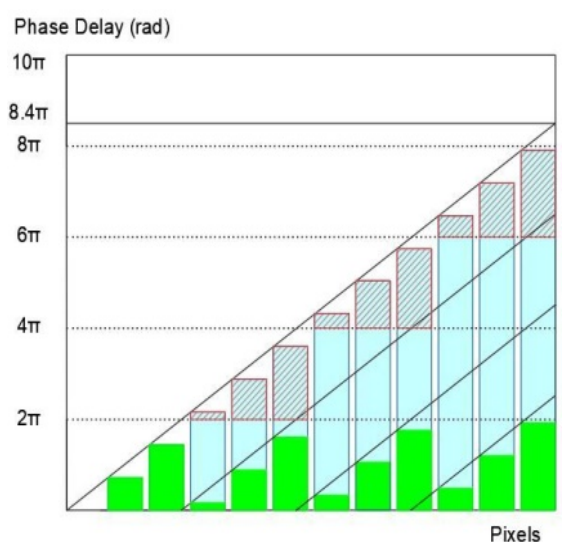

(b)

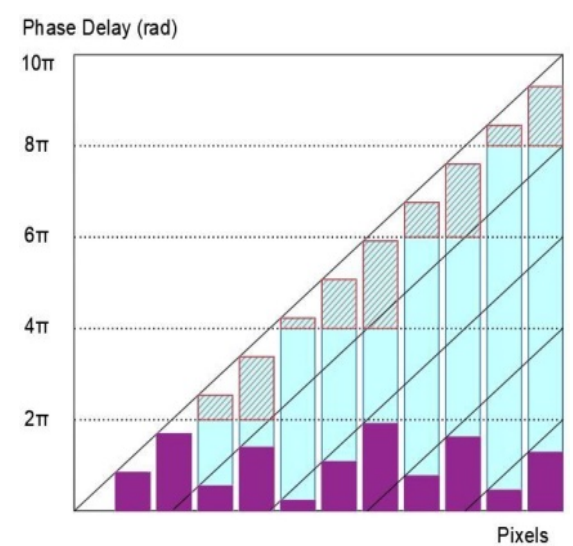

(c)

Figure 3. Schematic of the blaze grating structure over 12 pixels. The full colored orange, green, and purple rectangles present the wrapped phase delay of each pixel, corresponding to different integer and fractional topologies: (a) Topology +4 , (b) Topology +4.2 , (c) Topology +5 . In all pictures, the sign of the topology is positive, corresponding to a deviation to the right or up.

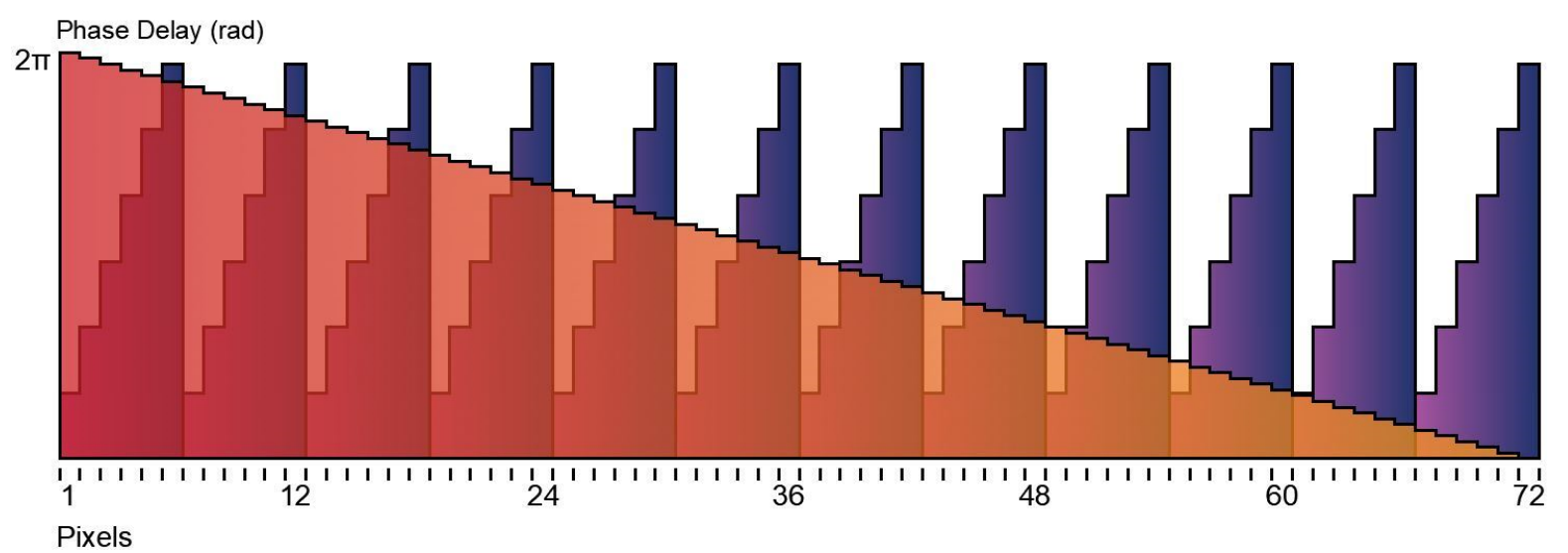

Figure 4. Schematic of the blaze grating for 72 pixels. The purple phase step structure corresponds with a topology +12 and the orange structure corresponds with a topology -1 .

The deviation angle, $\theta$, generated by the blaze is a function of its period and working wavelength [33]:

$$
\sin \theta=\frac{\lambda}{\Delta}
$$

Hence, the longer the wavelength or the shorter the period, the larger the diffraction angle, as long as the period is significantly larger than the wavelength. Additionally, the smaller the period, the smaller the pixels, in order not to compromise the diffraction efficiency, which is the real practical limiting factor in passive ITO based devices such as this one.

\subsection{Manufacturing of LC Cells}

The 1D liquid crystal cell is made up of an ITO coated glass sandwich that is filled up with nematic liquid crystal. One of the substrates is pixelated using a DLW technique, which allows generating high resolution structures in wafers with transparent ITO (Figure 5). The electrodes (pixels) are separated by less than $3 \mu \mathrm{m}$, leading to devices with a high fill factor [25]. The DLW installation (Lasing S.A.), consists of a UV laser with a CNC controlled XYZ-stage that shifts the substrate in XY while maintaining the focal distance using a second laser feedback system. 


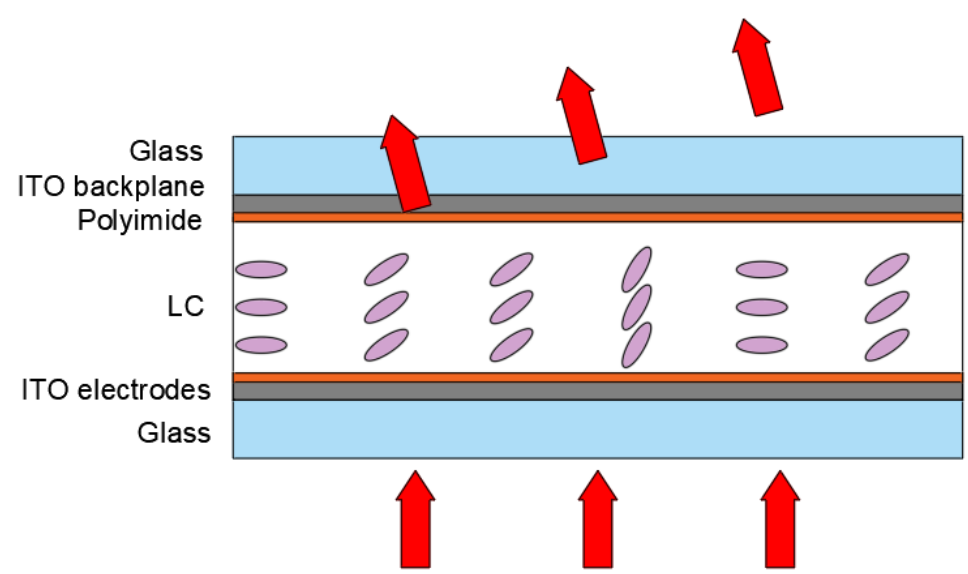

Figure 5. LC cell schematic, with their layer structure.

Both the pixelated and the continuous glass wafers were conditioned with rubbed polyimide PIA-2304(Chisso LIXON aligner, Tokyo, Japan), ensuring a uniform homogenous LC alignment. The cell thickness was controlled using randomly positioned $6.4 \mu \mathrm{m}$ diameter cylindrical silica spacers. Once the cell is mounted and sealed, it is filled in vacuum with the positive nematic high birefringence liquid crystal MDA-98-1600 (Merck KGaA, Darmstadt, Germany). The thickness of the cell and the birefringence determined at $632 \mathrm{~nm}$ of the LC allow for an optical retardation variation of $\Delta \mathrm{n}_{632 \mathrm{~nm}} \cdot \mathrm{L}=0.266 \cdot 6.4 \mu \mathrm{m}=1.7 \mu \mathrm{m}$, which means that, assuming that the reduction in birefringence does not exceed $10 \%$, the manufactured cell will be applicable to wavelengths close to $1550 \mathrm{~nm}$, which in turn would allow for twice the steering angle. The light transmission of the substrates limits the minimum working wavelength to about $400 \mathrm{~nm}$.

Interconnections between the employed flex connector and the ITO electrodes were done using an Anisotropic Conductive Adhesive (Hitachi Chemical, Tokyo, Japan). In the contact area, the ITO between electrodes was eliminated by multiple ablation lines.

The free end of the flex connector was connected directly to the driving electronics, providing full and independent control of each of the electrodes.

\subsection{The $2 D$ Device}

The 2D LC BS device is made up by cascading two orthogonal 1D liquid crystal cells. One is in charge of deviating the incident light beam in the azimuthal angle, while the other produces the deviation in the zenithal angle.

Both cells were manufactured following the same steps, and both were based on the same working principle of the LC. Each grating is made of 72 parallel pixels, with a $15 \mu \mathrm{m}$ pitch. Interpixel size is kept smaller than $3 \mu \mathrm{m}$, reducing any fringe effect. The resulting active area is $1.1 \times 1.1 \mathrm{~mm}^{2}$.

A CAD application was used to develop and design the pixel layout, including the active area and the fan-out (Figure 6). In the final cascaded configuration, the pixels of the two cells are perpendicular to each other in the active area. The rubbing direction, however, is common to both of the cells (Figure 7).

The incident light must be polarized in the same plane as the rubbing direction, determined in the manufacturing process. 


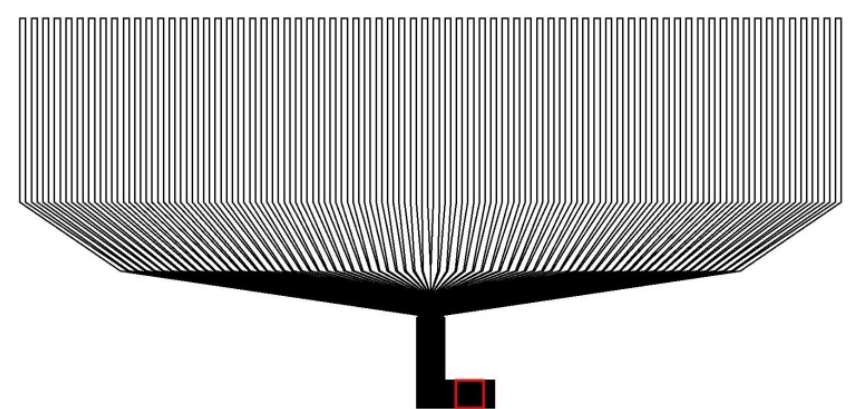

(a)

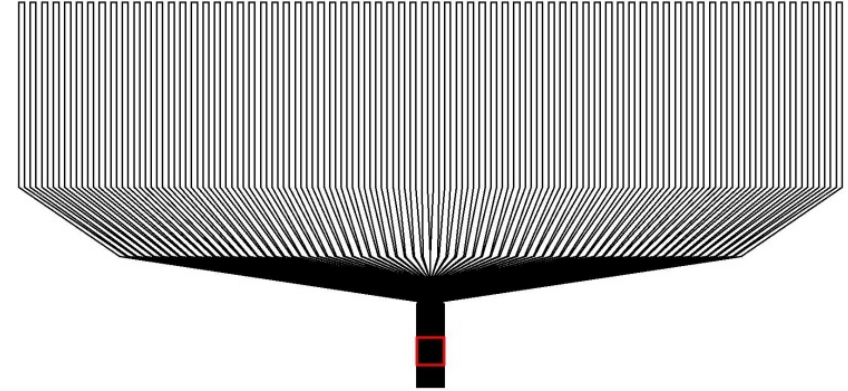

(b)

Figure 6. Pixel layout of the cells showing the contact fan-out. The active areas are marked with red squares. When overlapped, they create the 2D steering: (a) Horizontal pixels; (b) Vertical pixels.

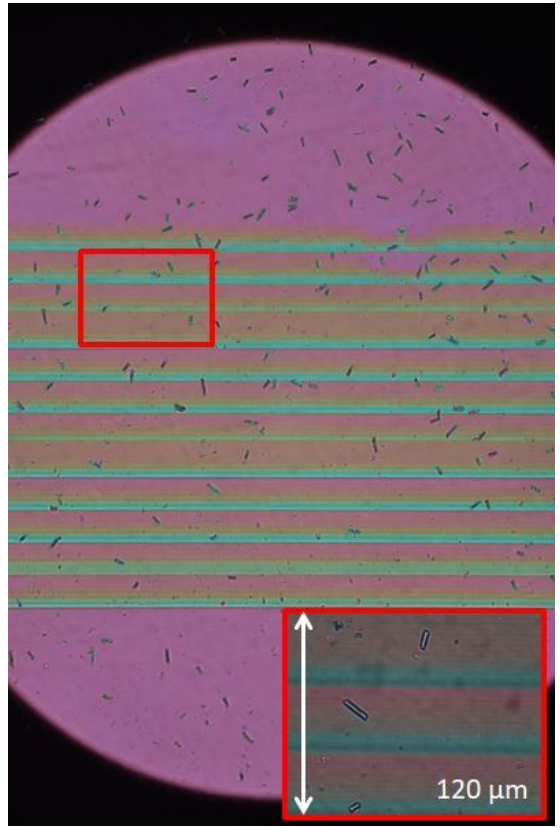

(a)

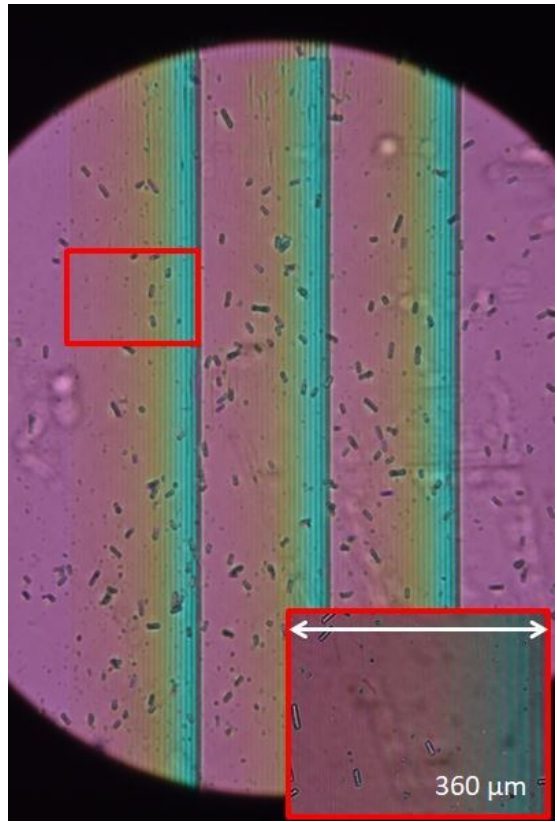

(b)

Figure 7. View of the active area in the microscope between cross polarizers: (a) Horizontal active area with topology +12 (pattern repetition for every 6 pixels, $90 \mu \mathrm{m}$ ); (b) Vertical active area with topology +3 (pattern repetition for every 24 pixels, $360 \mu \mathrm{m})$. Inserts show areas of $360 \times 120 \mu \mathrm{m}^{2}$, in which one may appreciate the individual pixels.

\subsection{Electronic Driver}

An electronic driver has been specially designed for individual electronic control of every pixel of the LC cell (Figure 8). It employs a 12 bits PWM signal for each of the pixels of the two LC cells. The electronic driver is addressed using a standard SPI protocol. An Arduino UNO board acts as a USB-SPI for the communication between the controlling computer and the LC cell driver.

The driver uses the 24 channel TLC5947 driver (PWM LED driver) from Texas Instrument (Dallas, TX, USA), with the output adjusted to TTL voltage levels by setting the control resistance to $18 \mathrm{k} \Omega$ and employing a $10 \mathrm{k} \Omega$ pull-up resistor. Each PWM voltage output is connected to the digital input of an analogue switch, ADG5413, from Analog Devices, which switches between an externally supplied AC signal and ground. The PWM cycle of approximately $1.3 \mu \mathrm{s}$ is sufficiently fast for the LC to perceive only the effective RMS signal of the supplied AC field, which is a $5 \mathrm{~V}_{\mathrm{pp}} 1 \mathrm{kHz}$ signal. 


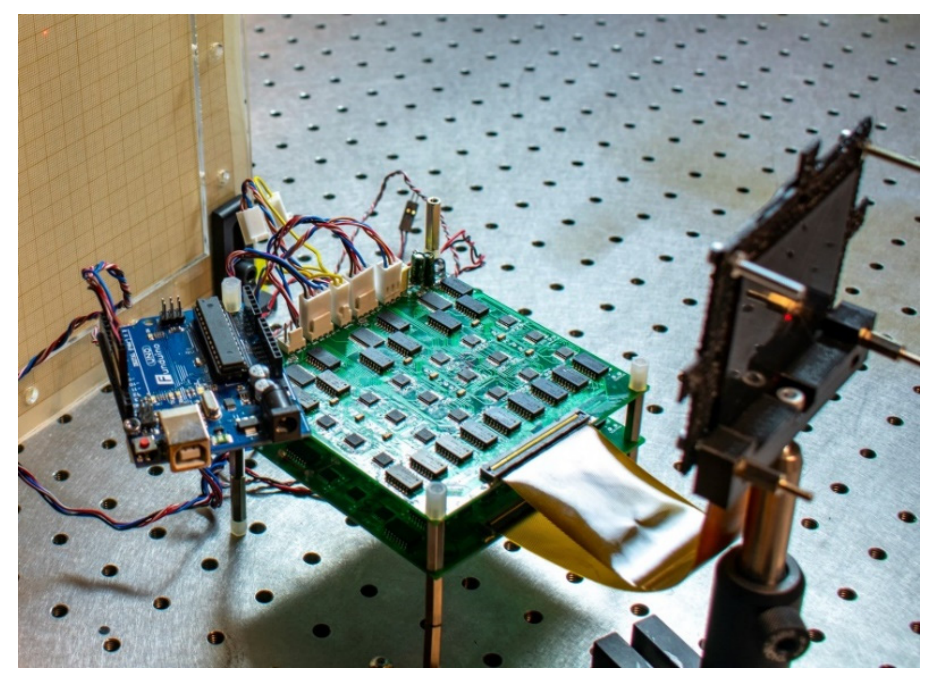

Figure 8. The 12 bits PWM driver that controls the LC cells.

The TLC5947 may be daisy chained. In the presented driver, three TLCs are combined per driver board, and two boards are daisy chained, providing individual control of all the $144(72+72)$ pixels in the two LC devices. The power consumption of the driver is less than $2 \mathrm{~W}$.

\subsection{Calibration}

The LC cells were calibrated at room temperature $\left(25^{\circ}\right)$ to establish their relationship between driving PWM signal and induced phase delay, as previously described [34] (Figure 9).

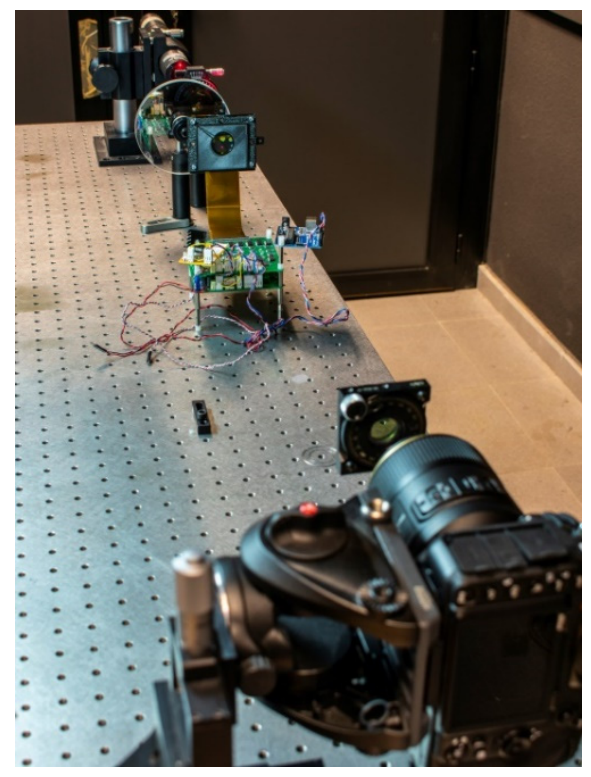

Figure 9. Calibration set up, including $632.8 \mathrm{~nm}$ He-Ne laser, attenuator, LC cells, electronic driver, polarizers, and camera.

The phase delay as a function of the PWM duty cycle (dc) follows a pseudo exponential decreasing function:

$$
\delta=A \cdot \mathrm{e}^{-B \cdot \mathrm{dc}}+C,
$$

The fitted intensity curve (Equation (2)) was employed to calculate the required PWM for any desired retardation level used in the different topologies (Figure 10). 


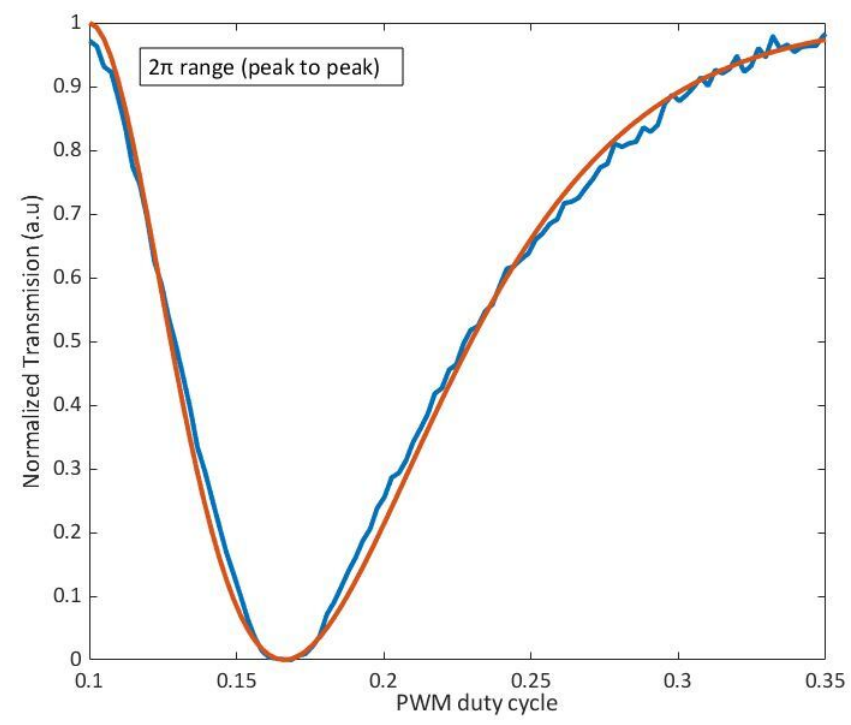

Figure 10. Normalized transmission intensity measured (blue) from the calibration, versus the fitted intensity (orange) as a function of the PWM duty cycle applied.

\section{Results and Discussion}

Figure 11 shows the diffraction pattern in the far field for different integer topologies. The same topology is applied to each LC cell, resulting in a diagonal deviation in the plane perpendicular to the propagation direction $(\mathrm{XY})$.

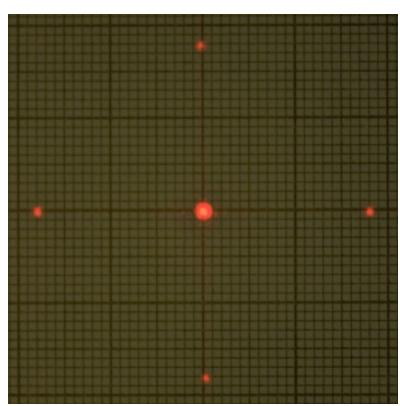

(a)

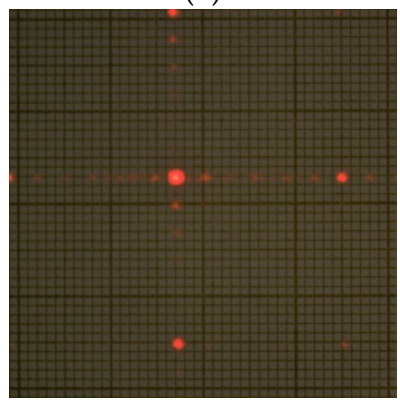

(e)

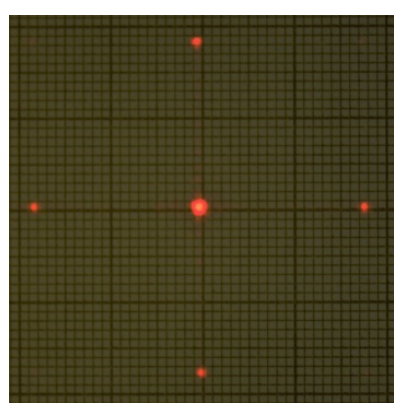

(b)

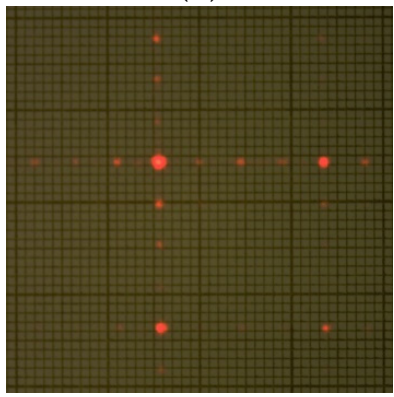

(f)

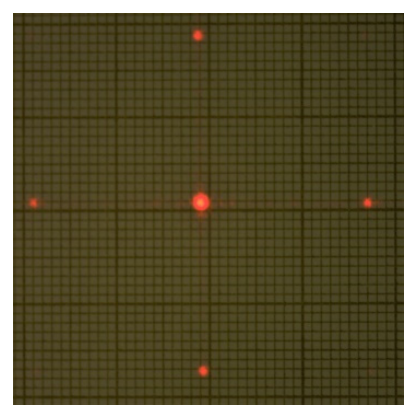

(c)

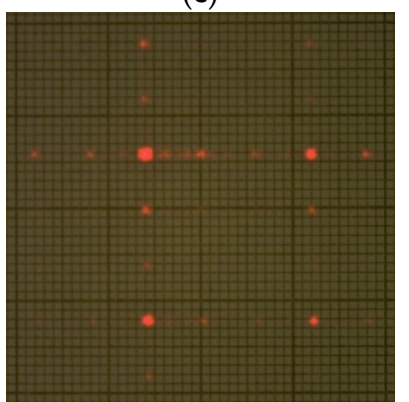

(g)

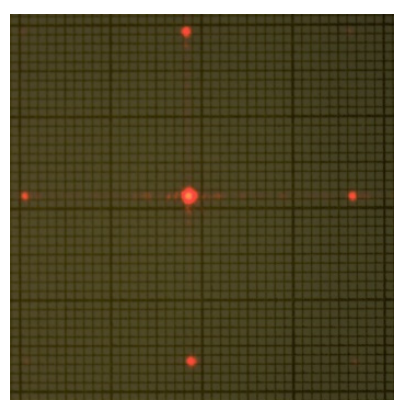

(d)

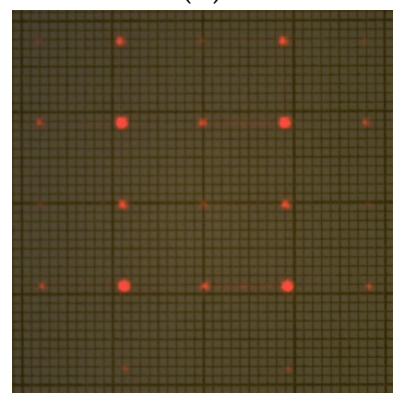

(h)

Figure 11. Representative photographs taken of the diffraction pattern for topologies applied in each cell. The distance between the LC BS and the screen is $\mathrm{z}=44 \mathrm{~cm}:(\mathbf{a}) 0 ;(\mathbf{b})+2 ;(\mathbf{c})+4 ;(\mathbf{d})+6 ;(\mathbf{e})+12 ;(\mathbf{f})+18 ;(\mathbf{g})+24 ;(\mathbf{h})+36$. Corresponding to diagonal deviation angles of: (a) $0^{\circ}$; (b) $0.065^{\circ}$; (c) $0.18^{\circ}$; (d) $0.27^{\circ}$; (e) $0.73^{\circ}$; (f) $0.83^{\circ}$; (g) $1.1^{\circ}$; (h) $1.65^{\circ}$.

It is clear from Figure 11 that the diffraction efficiency is best for smaller deviation angles and decreases as the diagonal deviation angle increases towards the maximum angle of 1.65 degrees. This is in accordance with diffraction theory, which predicts a $40 \%$ diffraction efficiency for a perfect 1D binary diffraction grating [23]. The almost perfect 
calibration of the system can be appreciated in the images of Figure 11 by the absence of the central 0 order peak, in all but the first OFF images.

The diffraction efficiency has been mapped (Figure 12a) for all combinations of integer topologies (i.e., where the 72 electrodes span an integer number of $2 \pi$ retardations) ranging from +36 to -36 in each cell. A photo of every configuration was taken, and the ratio between the intensity power of the deviated spot and the full frame was calculated afterwards. This procedure does ignore any power diffracted outside the imaged area but is insensitive towards intensity fluctuations in the laser light source. A conservative estimate of the background intensity reading, as well as occasional supersaturated camera pixels, may have led to a slight underestimate of the diffraction efficiency.

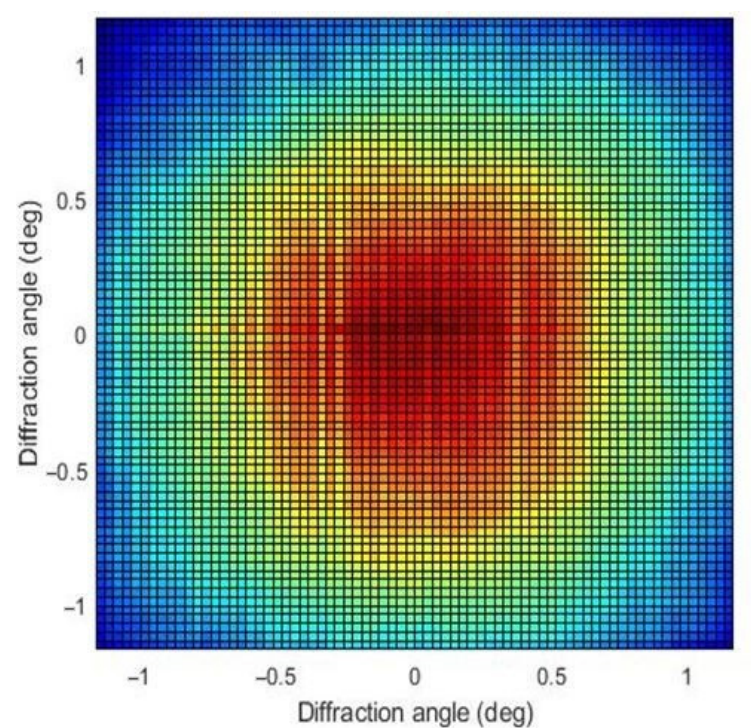

(a)

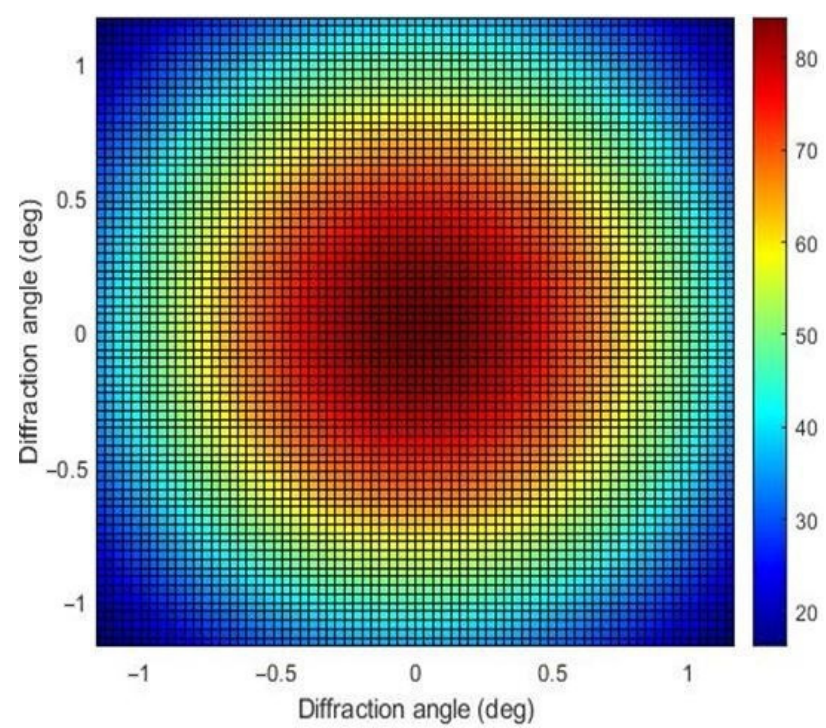

(b)

Figure 12. (a) Experimental $72 \times 72$ power diffraction efficiency map (\%) as a function of the diffraction angle. (b) Simulated $72 \times 72$ power diffraction efficiency map (\%) as a function of the diffraction angle.

In Figure 12a, one appreciates similar diffraction efficiency for both horizontal and vertical axes, indicating that the vertical rubbing direction along the electrode of one device, and perpendicular to the next device, is only marginally affecting the grating efficiency.

The beam steerer response has been simulated using Fast Fourier Transform of a simple step-like phase variation, with $25 \%$ transmission reduction in the interpixel space (Figure 12b) caused by the DLW. The simulated diffraction efficiency, using this simple model, has been mapped for all combinations of integer topologies ranging from +36 to -36. A 2D Fast Fourier Transform (2D FFT) is used to calculate the evolution of the electric field in the far field [35]:

$$
E_{\text {far field }}=X(\lambda, z) * 2 D \operatorname{FFT}\left(A_{P}\left(\varnothing_{\text {Total }}\right)\right),
$$

where $A_{P}(\varnothing)$ represents the aperture in the grating plane as a function of the phase profile. $X(\lambda, z)$ is a quadrature function with elements: the wave length of the incident laser beam $(\lambda)$, and the distance $(z)$ from the system to the screen.

Including crosstalk, fringe effects or higher order effects had no positive impact on the similarity between model and measurements. Similarly, the simple model coincides with observation of the absence of $X Y$ asymmetry, which could be assigned to the rubbing being either parallel or perpendicular to the pixels.

The PWM working range spans approximately a quarter of the full 12 bits PWM range, i.e., a 10 bits working resolution. Thus, an almost infinitely small angular step size may be used as a consequence of the full independence of each electrode (Figure 13). 


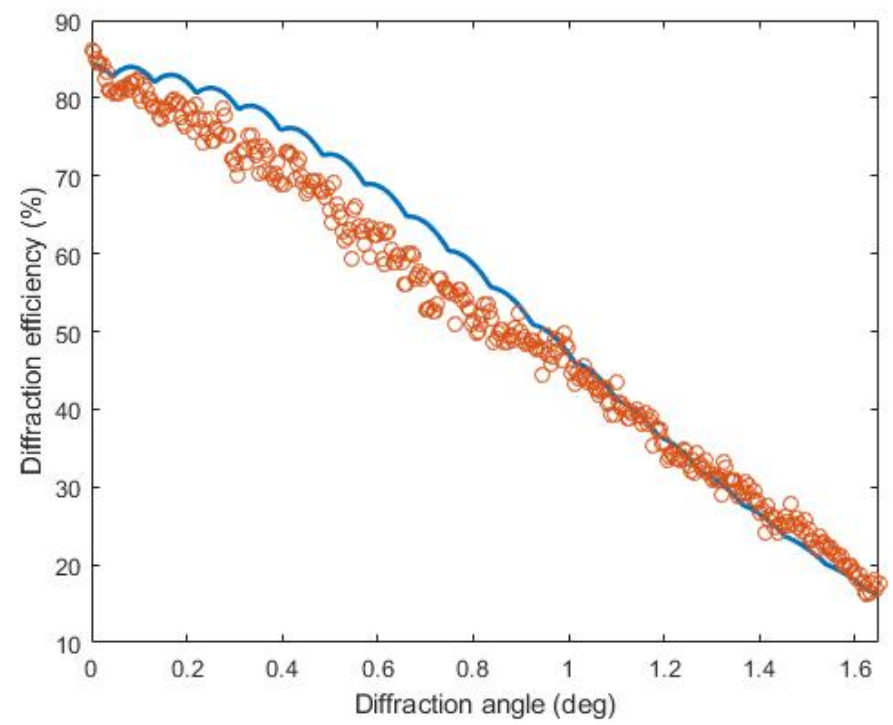

Figure 13. Fractional number topologies with a minimum step of 0.005 degrees has been measured in fractional micro steps of $0.1(0,+0.1,+0.2, \ldots,+36)$ along the diagonal in the XY plane. In blue is the simulated efficiency curve. In orange is the experimental efficiency map measured from topology 0 to +36 . A total of 361 points was analyzed, following the same procedure as in the previous experiment.

\section{Conclusions}

A 2D beam steering device has been demonstrated using passive electrodes and a novel electronic driver. An efficiency map of the device has been measured, where it is possible to study how the topology of the grating affects not only the deviation angle, but also the amount of light that is deviated in one direction. The use of a DLW technique to create the electrode pattern improves the efficiency, leading to a device with high fill factor. The phase variation of the interpixel gap has proven to be negligible in this device, possibly due to the small interpixel size. The maximum diagonal steering range for the binary pattern ( $16 \%$ diffraction efficiency) ranges from -1.65 to +1.65 degrees, and may be varied in steps of 0.005 degrees.

Author Contributions: Conceptualization, M.G.d.B. and M.A.G.; methodology, M.G.d.B., M.A.G., and X.Q.A.; software, M.G.d.B. and M.A.G.; validation, M.G.d.B., M.A.G., and X.Q.A.; investigation, M.G.d.B. and M.A.G.; writing—original draft preparation, M.G.d.B. and M.A.G.; writing—review and editing, J.M.O.; supervision, M.A.G. and X.Q.A. All authors have read and agreed to the published version of the manuscript.

Funding: This research was funded by the Spanish Ministerio de Economía y Competitividad through "Programa RETOS" ("Subtopic"-TEC2016-77242-C3-2-R) and by the EU structural funds (FEDER) and the Communidad de Madrid through the "Programa de Actividades de I + D" ("SINFOTON2CM"-S2018/NMT-4326) and the "Programa de Doctores Industriales" (IND2017/TIC-7835).

Institutional Review Board Statement: Not applicable.

Informed Consent Statement: Not applicable.

Data Availability Statement: All data are reported in the paper.

Conflicts of Interest: The authors declare no conflict of interest. The funders had no role in the design of the study; in the collection, analyses, or interpretation of data; in the writing of the manuscript, or in the decision to publish the results.

\section{References}

1. Hossack, W.J.; Theofanidou, E.; Crain, J.; Heggarty, K.; Birch, M. High-Speed Holographic Optical Tweezers Using a Ferroelectric Liquid Crystal Microdisplay. Opt. Express 2003, 11, 2053. [CrossRef]

2. Otón, E.; Pérez-Fernández, J.; López-Molina, D.; Quintana, X.; Otón, J.M.; Geday, M.A. Reliability of Liquid Crystals in Space Photonics. IEEE Photonics J. 2015, 7, 1-9. [CrossRef] 
3. Li, C.; Cao, X.; Wu, K.; Li, X.; Chen, J. Lens-Based Integrated 2D Beam-Steering Device with Defocusing Approach and Broadband Pulse Operation for Lidar Application. Opt. Express 2019, 27, 32970. [CrossRef] [PubMed]

4. Lin, Y.; Ai, Y.; Shan, X.; Liu, M. Simulation of Two-Dimensional Target Motion Based on a Liquid Crystal Beam Steering Method. Opt. Eng. 2015, 54, 056102. [CrossRef]

5. Hellman, B.; Luo, C.; Chen, G.; Rodriguez, J.; Perkins, C.; Park, J.-H.; Takashima, Y. Single-Chip Holographic Beam Steering for Lidar by a Digital Micromirror Device with Angular and Spatial Hybrid Multiplexing. Opt. Express 2020, 28, 21993. [CrossRef] [PubMed]

6. He, Z.; Gou, F.; Chen, R.; Yin, K.; Zhan, T.; Wu, S.-T. Liquid Crystal Beam Steering Devices: Principles, Recent Advances, and Future Developments. Crystals 2019, 9, 292. [CrossRef]

7. Wang, Y.; Zhou, G.; Zhang, X.; Kwon, K.; Blanche, P.-A.; Triesault, N.; Yu, K.; Wu, M.C. 2D Broadband Beamsteering with Large-Scale MEMS Optical Phased Array. Optica 2019, 6, 557. [CrossRef]

8. Seo, Y.-H.; Hwang, K.; Kim, H.; Jeong, K.-H. Scanning MEMS Mirror for High Definition and High Frame Rate Lissajous Patterns. Micromachines 2019, 10, 67. [CrossRef] [PubMed]

9. Yoo, B.-W.; Megens, M.; Chan, T.; Sun, T.; Yang, W.; Chang-Hasnain, C.J.; Horsley, D.A.; Wu, M.C. Optical Phased Array Using High Contrast Gratings for Two Dimensional Beamforming and Beamsteering. Opt. Express 2013, 21, 12238. [CrossRef] [PubMed]

10. Yang, J.; Su, X.; Xu, P.; Gu, Z. Beam Steering and Deflecting Device Using Step-Based Micro-Blazed Grating. Opt. Commun. 2008, 281, 3969-3976. [CrossRef]

11. Römer, G.R.B.E.; Bechtold, P. Electro-Optic and Acousto-Optic Laser Beam Scanners. Phys. Procedia 2014, 56, 29-39. [CrossRef]

12. Xu, J.; Cua, M.; Zhou, E.H.; Horie, Y.; Faraon, A.; Yang, C. Wide-Angular-Range and High-Resolution Beam Steering by a Metasurface-Coupled Phased Array. Opt. Lett. 2018, 43, 5255. [CrossRef] [PubMed]

13. Niu, Q.; Wang, C. High Precision Beam Steering Using a Liquid Crystal Spatial Light Modulator. Opt. Quant. Electron. 2019, 51, 180. [CrossRef]

14. Kim, Y.; Won, K.; Kim, Y.; An, J.; Song, H.; Kim, S.; Choi, C.-S.; Lee, H.-S. Electrically Tunable Transmission-Type Beam Deflector Using Liquid Crystal with High Angular Resolution. Appl. Opt. 2018, 57, 5090. [CrossRef] [PubMed]

15. Zohrabi, M.; Cormack, R.H.; Gopinath, J.T. Wide-Angle Nonmechanical Beam Steering Using Liquid Lenses. Opt. Express 2016, 24, 23798. [CrossRef]

16. McManamon, P.F.; Bos, P.J.; Escuti, M.J.; Heikenfeld, J.; Serati, S.; Xie, H.; Watson, E.A. A Review of Phased Array Steering for Narrow-Band Electrooptical Systems. Proc. IEEE 2009, 97, 1078-1096. [CrossRef]

17. Yousefzadeh, C.; Van Rynbach, A.; Bos, P.J. Design of a Large Aperture, Tunable, Pancharatnam Phase Beam Steering Device. Opt. Express 2020, 28, 991. [CrossRef]

18. Yang, H.; Chu, D.P. Digital Phase-Only Liquid Crystal on Silicon Device with Enhanced Optical Efficiency. OSA Contin. 2019, 2, 2445. [CrossRef]

19. Zhang, Z.; You, Z.; Chu, D. Fundamentals of Phase-Only Liquid Crystal on Silicon (LCOS) Devices. Light Sci. Appl. 2014, 3, e213. [CrossRef]

20. Yin, K.; Lee, Y.-H.; He, Z.; Wu, S.-T. Stretchable, Flexible, Rollable, and Adherable Polarization Volume Grating Film. Opt. Express 2019, 27, 5814. [CrossRef]

21. Kim, J.; Oh, C.; Escuti, M.J.; Hosting, L.; Serati, S. Wide-Angle Nonmechanical Beam Steering Using Thin Liquid Crystal Polarization Gratings; Gonglewski, J.D., Carreras, R.A., Rhoadarmer, T.A., Eds.; SPIE: San Diego, CA, USA, 2008; p. 709302.

22. Yin, K.; Zhan, T.; Xiong, J.; He, Z.; Wu, S.-T. Polarization Volume Gratings for Near-Eye Displays and Novel Photonic Devices. Crystals 2020, 10, 561. [CrossRef]

23. Otón, J.M.; Otón, E.; Quintana, X.; Geday, M.A. Liquid-Crystal Phase-Only Devices. J. Mol. Liq. 2018, 267, 469-483. [CrossRef]

24. Yin, K.; Xiong, J.; He, Z.; Wu, S.-T. Patterning Liquid-Crystal Alignment for Ultrathin Flat Optics. ACS Omega 2020, 5, 31485-31489. [CrossRef] [PubMed]

25. Zola, R.S.; Bisoyi, H.K.; Wang, H.; Urbas, A.M.; Bunning, T.J.; Li, Q. Dynamic Control of Light Direction Enabled by StimuliResponsive Liquid Crystal Gratings. Adv. Mater. 2019, 31, 1806172. [CrossRef]

26. Shang, X.; Cuypers, D.; Baghdasaryan, T.; Vervaeke, M.; Thienpont, H.; Beeckman, J.; Neyts, K.; Li, Q.; Wu, C.; Li, H.; et al. Active Optical Beam Shaping Based on Liquid Crystals and Polymer Micro-Structures. Crystals 2020, 10, 977. [CrossRef]

27. Oton, E.; Morawiak, P.; Mazur, R.; Quintana, X.; Geday, M.A.; Oton, J.M.; Piecek, W. Diffractive and Refractive Liquid Crystal Devices Based on Multilayer Matrices. J. Lightwave Technol. 2019, 37, 2086-2093. [CrossRef]

28. Lee, H.; Kim, H.; Yang, S.; Hwang, J.; Lee, J.-H.; Choi, J. Electro-Optical Properties of a Nematic Liquid Crystal Aligned with a Mixture of Nanofibres and Polyimide. J. Phys. D Appl. Phys. 2014, 47, 345303. [CrossRef]

29. Bennis, N.; Geday, M.; Quintana, X.; Cerrolaza, B.; Medialdea, D.; Spadło, A.; Dąbrowski, R.; Otón, J. Nearly-Analogue Blazed Phase Grating Using High Birefringence Liquid Crystal. Opto Electron. Rev. 2009, 17. [CrossRef]

30. Xu, L.; Zhang, J.; Wu, L.Y. Influence of Phase Delay Profile on Diffraction Efficiency of Liquid Crystal Optical Phased Array. Opt. Laser Technol. 2009, 41, 509-516. [CrossRef]

31. Moser, S.; Ritsch-Marte, M.; Thalhammer, G. Model-Based Compensation of Pixel Crosstalk in Liquid Crystal Spatial Light Modulators. Opt. Express 2019, 27, 25046. [CrossRef] [PubMed]

32. Kim, Y.; Won, K.; An, J.; Hong, J.-Y.; Kim, Y.; Choi, C.-S.; Song, H.; Song, B.; Suk Kim, H.; Bae, K.-D.; et al. Large-Area Liquid Crystal Beam Deflector with Wide Steering Angle. Appl. Opt. 2020, 59, 7462. [CrossRef] [PubMed] 
33. Wang, X.; Wu, L.; Li, M.; Wu, S.; Shang, J.; Qiu, Q. Theoretical and Experimental Demonstration on Grating Lobes of Liquid Crystal Optical Phased Array. Int. J. Opt. 2016, 2016, 1-6. [CrossRef]

34. Caño-García, M.; Quintana, X.; Otón, J.M.; Geday, M.A. Dynamic Multilevel Spiral Phase Plate Generator. Sci. Rep. $2018,8$. [CrossRef] [PubMed]

35. Goodman, J.W. Introduction to Fourier Optics, 4th ed.; W.H. Freeman: New York, NY, USA, 2017; pp. 130-160. 